\title{
Thumbs up and down
}

$\mathrm{O}$ verreliance on small handheld devices may be predisposing the current generation of electronics addicts to early development of osteoarthritis and other potentially chronic joint conditions such as "BlackBerry thumb," some physiotherapists say.

BlackBerry thumb is a repetitive strain injury that causes tendons at the base of the thumb to become inflamed as a consequence of hyperextension and awkward positioning, as can occur during extended use of a BlackBerry or other smartphone.

Injuring a thumb can have a substantial impact because a person's "thumb is responsible for $60 \%$ of the functioning of their hand," says Dolores Langford, a registered physiotherapist and certified hand therapist in West Vancouver, British Columbia.

The size and weight of hand-held phones mislead people about the pressure the devices require, she says. "If you're texting, the pressure at the tip of your thumb is amplified twelve times by the time it gets to the base of your thumb," so the basal joint has to absorb that shock with every keystroke.

Youngsters “don't phone each other anymore. Everything is done via text. I have a teenage daughter and I'm seeing in her friends that they are also starting to complain of pain in their thumbs," Langford adds. "There's just so much pressure on their little hands that I think that down the line, in 15, 20 years, we're going to see some of the repercussions of this."

Langford frequently sees patients with flare-ups in the base of their thumbs who must give up texting for an extended period to reduce pain.

But others appear skeptical that there is such a condition as BlackBerry thumb or that it is strictly associated with the use of hand-held devices.

"As much as it is more commonly known as 'BlackBerry thumb,' the majority of people we see don't actually get it from using a BlackBerry, which is

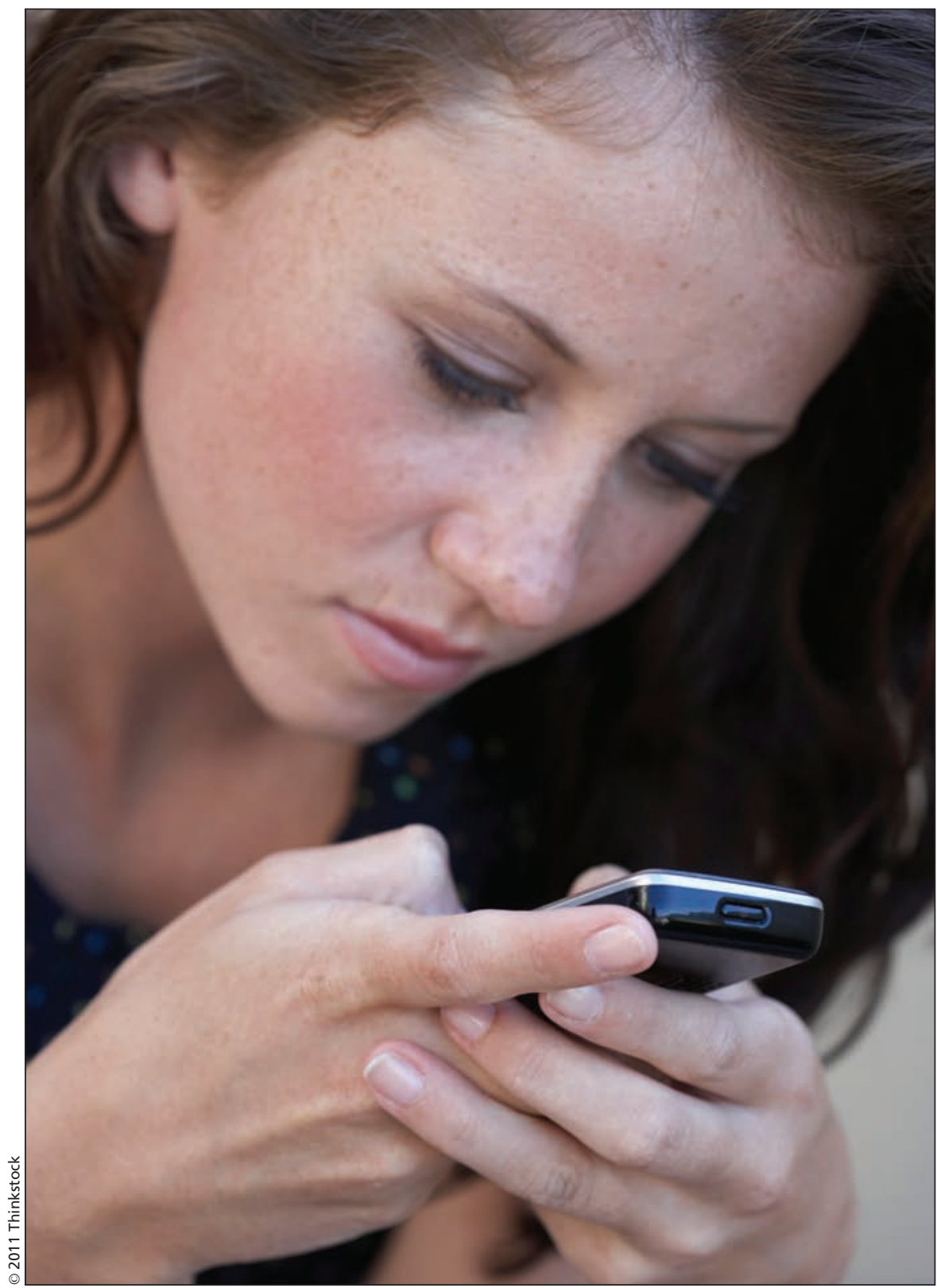

Some teenagers who spend hours texting friends every week already have pain in their thumbs and may one day end up with other health repercussions from the repetitive strain on their hands, say some physiotherapists and hand therapists.

a bit odd," says Joseph Federico, a registered physiotherapist who operates a clinic in Ottawa, Ontario.

It's just a fad name for an injury that already exists, he says, adding that the condition has been called "mommy thumb" by some, but that the technical name is deQuervain's tenosynovitis.
It's very common in mothers of toddlers because they spread their thumbs away from the rest of their fingers to cover more surface area when picking up their children, Federico says. It's also seen sometimes in people with hobbies, such as making crafts that require intricate hand work. 
One study on whether there is a link between use of hand-held devices and pain indicated that the consequences often extend well beyond the thumb alone (Appl Ergo 2011;42:371-8)

In frequent users of hand-held devices, severe pain was felt primarily in the neck, upper back and shoulders, often requiring painkillers and becoming quite disabling, says Richard Wells, director the Centre of Research Expertise for the Prevention of Musculoskeletal Disorders in Waterloo, Ontario. "If we're creating a lot of 17year-olds, 19-year-olds who have more chronic shoulder, neck and arm problems, then they got a lifetime of problems ahead of them."

Wells adds that while the study involved only 140 university students, faculty and administrative staff, the findings suggest that the degree of pain rises with the frequency of use of mobile devices for such activities as texting, emailing, Internet browsing, talking on the phone and gaming. For example, pain in the right shoulder after 4.09 hours of gaming was reported to be as high as 9.42 (out of ten) on a severity index. It was reported at 8.33 after 3.76 hours, indicating that after a period of time, relatively small incremental increases in period of usage resulted in higher levels of severity of pain.
The next step will involve determining whether the type and shape of mobile devices (such as slide phones, flip phones and touch screens) affects the degree of strain or damage to people's thumbs.

Until the findings of such research is conclusive, Wells says, people should try to minimize the potential damage that mobile device use can cause by, for example, resting their arms on a table while using the devices to protect their shoulders and neck.

It's all about ergonomics, says Federico. "If you use the device in a more ergonomically correct way, it's going to minimize your risk for injury. ... Try to keep your wrist in a more neutral position so that the wrist is not cocked up towards you." He also recommends stretching exercises, such as the Finkelstein test - point thumb at ceiling and fingers forward; place thumb in palm of hand; wrap fingers around thumb; bend wrist toward ground - after prolonged periods of mobile device use.

That's how physiotherapists actually diagnose deQuervain's tenosynovitis, he adds. "If you have pain with that, then we say, 'Oh, that's a positive Finkelstein test, you have BlackBerry thumb'.'

Although Federico believes the actual incidence of the disorder as a consequence of hand-held device use is rare, that hasn't stopped law firms such as
Roberts Jackson Ltd. in the United Kingdom from surmising that employees will soon be seeking legal redress for having to use mobile devices, according to recent news reports (www.telegraph .co.uk/technology/BlackBerry/8403978 /BlackBerry-thumb-is-new-health-hazard .html). "Employers can expect a series of lawsuits from staff claiming compensation," the firm believes.

There's no evidence of similar legal action in Canada, although the Canadian Union of Public Employees says it is now working with the Canadian Standards Association on a general ergonomic standard for hand-held device use that is expected to be published within six to eight months, says Anthony Pizzino, national director of research, job evaluation and health and safety for public employees.

Although it's not expected that the voluntary standard will cover all mobile devices, it should introduce a preventive framework that allows manufactures and workplaces to adopt methods of recommended usage similar to those now advised for computer keyboards, says Pizzino. "We're looking at similar processes in terms of usage, amount of time that you use and design itself." Erin Walkinshaw, Ottawa, Ont.

CMAJ 2011. DOI:10.1503/cmaj.109-3911 